\title{
Design and Practice of Surveying Experiment System based on a Virtual Platform
}

\author{
https://doi.org/10.3991/ijet.v12i04.6924 \\ Caiyun Gao \\ Henan University of Urban Construction, Pingdingshan, China \\ gaocaiyungao@ $163 . \mathrm{com}$ \\ Qiutang $\mathrm{Wu}$ \\ Basic Surveying and Mapping Institute of Jiangxi Province, Nanchang, China \\ $249630128 @$ qq.com
}

\begin{abstract}
The experimental teaching of surveying is an effective method for students to acquire basic surveying skills and solve practical problems. By contrast, the traditional experimental teaching mode of surveying presents students with insufficient experimental opportunities and difficulties to achieve independent learning and interactive operation due to limitations in the allotted time for experiments, sites, instruments, and external environmental conditions. This study developed a virtual experimental system by combining network virtual reality technology and surveying experiment. The proposed system intends to provide students with additional experimental opportunities to achieve computer desktop-like virtual experimental devices and engineering construction scenes, thereby accomplishing the indoor simulation of field surveying. This virtual surveying experimental system mainly comprised four modules, namely, classroom teaching, classroom practice, teaching practice, and production practice. This system realized equipment, operation, and data virtualizations, as well as various highly realistic measurement experiments, such as topographic surveying, engineering setting out, and road surveying. Application results demonstrated that the proposed system was convenient for the independent study of students, stimulates their enthusiasm for learning, strengthens their operational ability, and achieves good teaching effect. Furthermore, this system provides a novel experimental teaching mode for surveying courses.
\end{abstract}

Keywords—surveying experiment, virtual reality, application

\section{$1 \quad$ Introduction}

The surveying course has strong practicality, applicability, and engineering quality. Students in this course are required not only to master the theoretical knowledge of surveying but also to comprehend operative skills of all kinds of instruments of surveying. In doing so, they can accomplish field measurement tasks quickly, proficiently, and independently. However, many universities still adopt the combined teaching mode of theoretical classroom teaching and outdoor practical teaching in the survey- 
ing course. In the teaching field, teachers demonstrate the basic operation procedure of surveying instruments to students, and then students implement the operation through training. Scarcity in experimental site, experimental apparatus, and external environment limits the practical opportunities of students, thereby hindering the training of their operation and application skills. Under such circumstance, new reforms to training such abilities of students can be introduced, such as providing computer desktop-like virtual experimental environments and instruments for indoor field operation training [1-2].

The progress of society has facilitated the rapid development of scientific technologies and the emergence of new teaching media, such as virtual reality (VR). VR integrates several information technologies, such as computer graphics, digital image processing, multimedia technology, and sensor technology [3-4]. The threedimensional representation and natural man-machine interaction environment of VR convey a sense of presence to users. Combining VR with multimedia teaching and network can develop a novel and effective teaching mode that can represent contents that cannot be fully expressed by traditional media.

Virtual simulation experimental teaching is currently widely applied in different majors in developed countries, such as micro-electronics online laboratory in Massachusetts Institute of Technology, surgery simulation laboratory of North Carolina University, virtual physics laboratory of University of Houston, and dynamic fluid virtual simulation experimental system of George Mason University [1]. However, relatively few majors in China, especially surveying engineering. To solve the problem above, this study developed a virtual experimental system by combining network virtual reality technology and surveying experiment.

\section{State of art}

The rapid development of surveying technologies in recent years has significantly diminished the period of acquiring updated knowledge. Given the continuous progress of experimental methods and technologies, the experimental teaching of surveying based on virtual technology has attracted considerable attention from Chinese and foreign scholars. The key to the experimental virtual teaching of surveying depends upon the essential instruments, outdoor measurement environment, and engineering environment. Tian Peng et al. realized virtual operation in understanding the total station electronic distance measuring device, arrangement, and basic measurement using the 3D Studio Max (3DMAX) software [2]. Koncny realized terrain environment simulation based on virtual technology [3]. Zhou Min et al. used the study of Tian Peng as basis to further enhance the virtual operation of the total station electronic distance measuring device, as well as expand virtual surveying equipment into the level, theodolite, and Global Positioning System (GPS) receiver, among others [5]. Deng Xingsheng et al. developed an experimental photogrammetry teaching system based on MATLAB/Simulink [6]. Wang Shitai et al. realized the scene virtualization of indoor and outdoor experimental environments based on virtual technology [7]. 
The results of the preceding research indicate that several existing studies on the experimental teaching of surveying based on virtual technology still possess the following disadvantages.

1. This process merely involves single virtual equipment and simple contents to fulfill the multiple-device requirements of an experimental surveying system.

2. The developed experimental virtual system mainly focuses on basic surveying experiment training and lacks a 3D scene display.

3. The existing experimental virtual systems mainly focus on one specific project (e.g., unmanned aerial photogrammetry), which presents difficulty in satisfying the internship requirements of surveying engineering, photogrammetry and remote sensing, and geographic information system (GIS) majors.

Hence, an experimental virtual surveying system with multiple pieces of equipment, as well as multiple scenes and projects, was developed using a virtual reality (VR) modeling language. This system mainly comprised four modules, namely, classroom teaching, classroom practice, teaching practice, and production practice. Furthermore, this system realized equipment, operation, and data virtualization, as well as real simulation of large engineering projects.

The remainder of this work is structured as follows. Section 3 establishes a virtual surveying experimental system. The implementation results of virtual surveying experimental system are reported in Section 4. Finally, the concluding remarks are provided in Section 5.

\section{Establishment of the virtual surveying experimental system}

\subsection{Requirements}

1. Close relation to textbook: The virtual surveying experimental system will be combined with the teaching content of each chapter of a textbook to accomplish substantial operation training of surveying instruments.

2. Sharing capability: For the convenience of spreading and sharing of education resources, the software will be operated on the Internet.

3. High universality: The developed virtual instrument model will be applied in different scenes. Given that the software contains many virtual models, a virtual instrument library will be constructed for unified management of these instruments. Accordingly, they can be applied in different virtual cases and can increase the number of new instrument models.

4. Good operation: The data size of constructed scene should not be too large to ensure smooth software operation on the Internet.

\subsection{Overall structure}

The virtual surveying experimental system is composed of four modules: classroom teaching, classroom practice, teaching practice, and production practice. Class- 
room teaching enables mastering of basic principles and operation flows of surveying instruments. Classroom practice allows mastering of operation skills of equipment, thereby helping in conducting basic surveying and surveying experiment. Teaching practice provides targeted training of specialized courses. Production practice offers training of application ability of comprehensive technologies and can establish a comprehensive training system of multiple devices, scenes, and projects based on virtual technologies, the system therefore overcomes the limitations of traditional measurement courses in equipment, sites, and environment [5]. The structure of the virtual surveying experimental system is shown in Fig. 1.

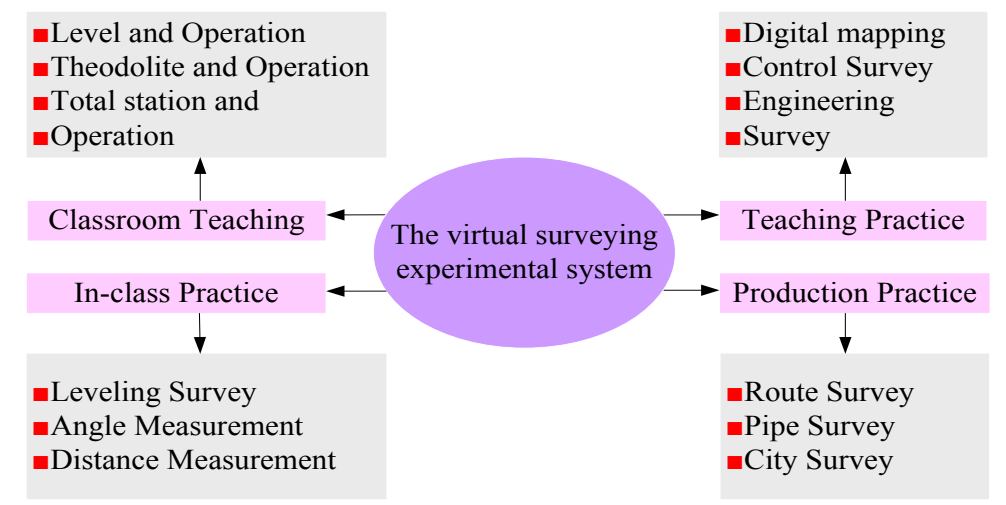

Fig. 1. Structure of virtual surveying experimental system

\subsection{Realization of the virtual surveying experimental system}

The virtual reality modeling language (VRML) was used for three-dimensional modeling of surveying instruments and scene development. The VRML document is generally composed of file header, node, and routes. Node is the basic unit of VRML document. The common version is VRML-pad2.1.exe editor and CosmoPlayer3.0 browser made by Parallel Graphics Company.

Virtual realization of surveying environment and instruments: Indoor surveying environment includes indoor constructions and indoor ancillary facilities, such as desks, doors, and windows. Consequently, a teaching environment for virtual indoor measurement training is provided to students. The outdoor surveying environment includes sky, terrains, surveying fields, and outdoor trees. An accessible environment for virtual outdoor measurement training is thus provided to students [8-9]. The virtual surveying instruments mainly include electronic theodolite, double frequency GPS receiver, gravity meter, foot stool of instruments, and prisma. Students can place measurement instruments in the virtual environment for operation training. The design sketch and operation interface of the virtual total station device are shown in Fig. 2. Students can click buttons on the operation interface using the mouse and then the panel screen will display the corresponding operation view [10]. The operation panel of this virtual device is made in accordance with the operation panel of real instrument. Students can click the instrument panel, control the telescope, and identify the 
accurate observation goal using the mouse, thereby accomplishing basic operation training of the total station device .

Virtual observation of angle and distance: Angle is observed by setting the rotation angles of the object on the current horizontal or vertical direction and through conversion from degree to radian. The measurement results will be displayed on the virtual liquid crystal display. Distance is measured by the ray collision detection principle. The ray is an endless line from one point in the world toward one direction. In the emission track, this point will stop emission once it collides with other models. Then, whether the ray is emitted to an object can be determined, thereby acquiring the object information.

Virtual realization of typical topographic surveying: After accomplishing the virtual operation of surveying instruments, students can learn through the operation module of virtual engineering to further understand the applications of surveying instruments in actual surveying projects. The construction scanning measurement based on a virtual 3D laser scanner is shown in Fig. 3. Topographic surveying of virtual GPS technology is shown in Fig. 4. The deformation monitoring of a virtual tunnel is shown in Fig. 5.

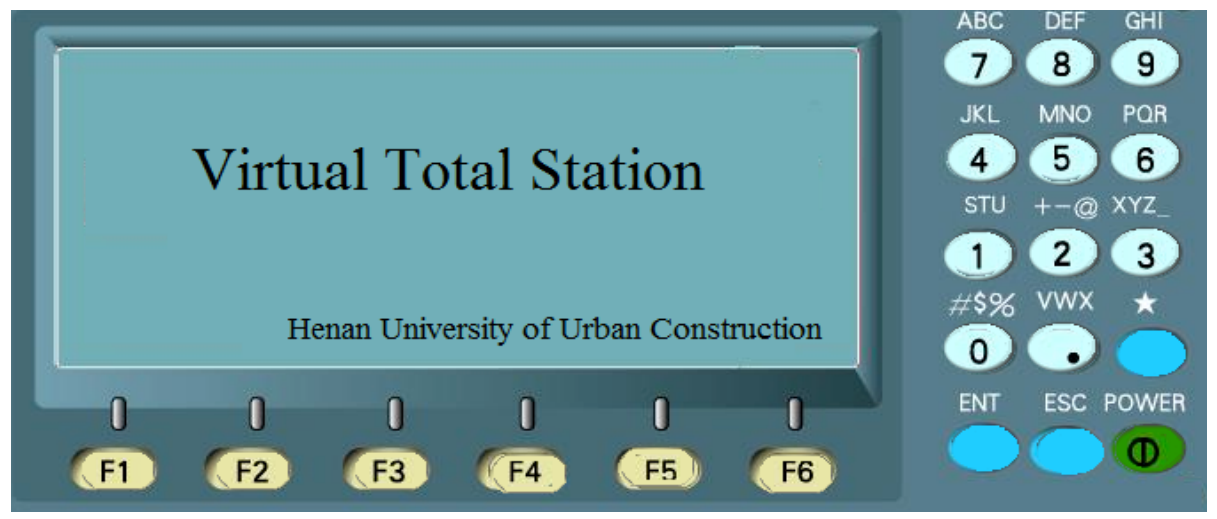

Fig. 2. Operation interface of virtual total station

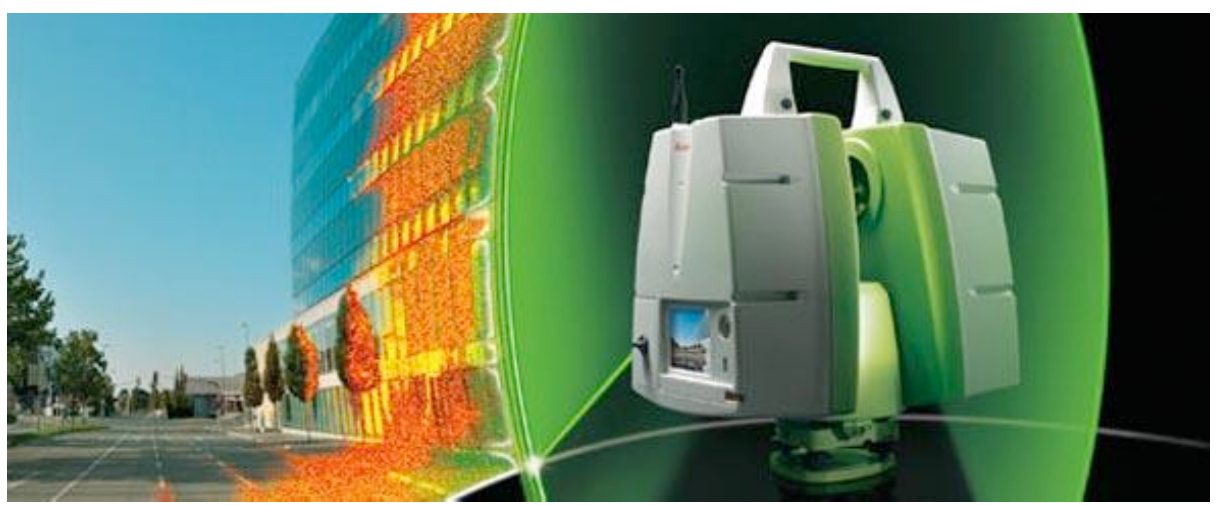

Fig. 3. Construction scanning measurement based on a virtual 3D laser scanner 


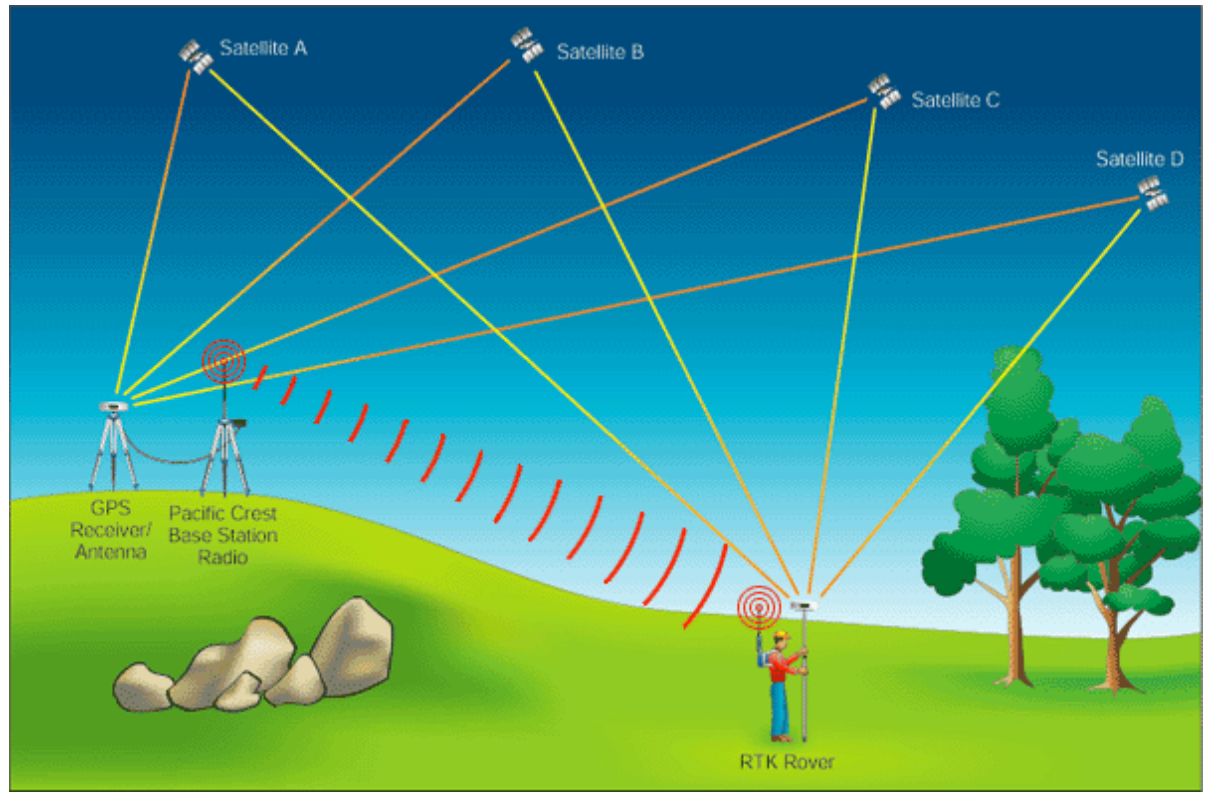

Fig. 4. Topographic surveying of virtual GPS technology

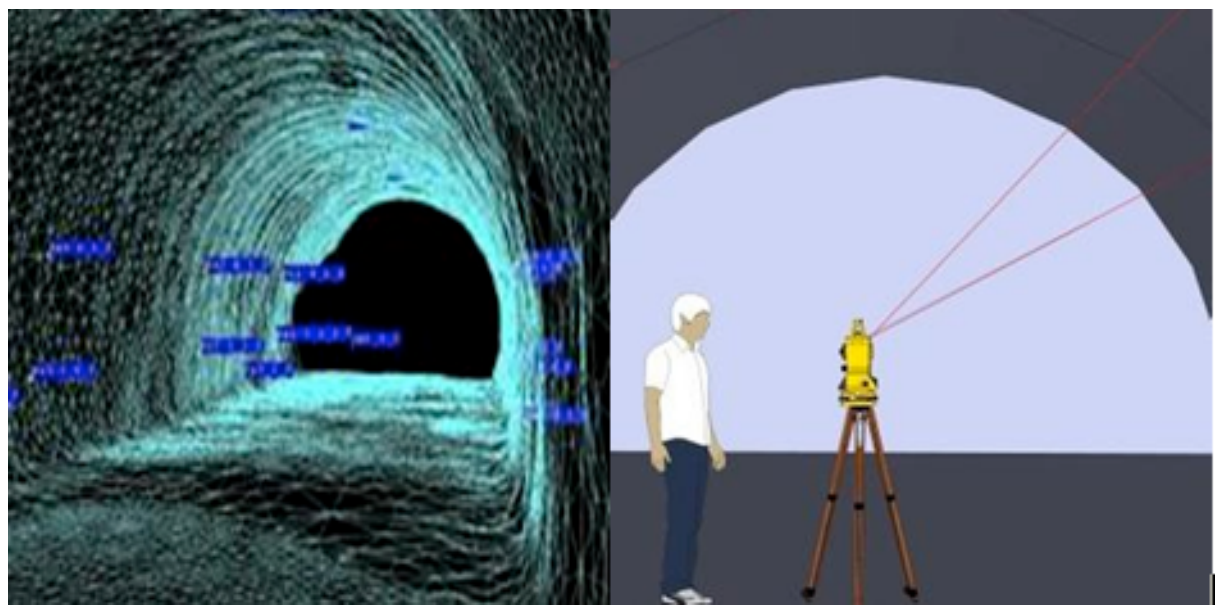

Fig. 5. Deformation monitoring of a virtual tunnel

\section{$4 \quad$ Experience of virtual surveying experimental system}

\subsection{Application of virtual surveying experimental system}

The virtual surveying experimental system can be beneficial in the following five aspects in surveying courses. 
1. Online teaching program: Teachers can upload teaching videos to the server and students can watch them online. Teaching contents will be contained in electronic textbooks, which can be downloaded by students for their autonomous learning.

2. Available operation of virtual instruments: After logging in the virtual experimental system, students can choose their desired instruments by use of the mouse and can exercise operation procedures of the instruments through the simulation operation panel. Students can also perform virtual measurement exercises based on the indoor and outdoor measurements of the system [11].

3. Increased experimental exercises: In consolidating students' learning effect, the virtual experimental system network increases the experimental exercises for students' autonomous selection of online answering and online practices.

4. Increased design and engineering experiments: Measurement courses involve many design and engineering experiments. Teachers design engineering experiments that enable students to develop measurement design in indoor and outdoor virtual environments, such as selection of measurement points and establishment of the measurement plan. The design results are then uploaded.

5. Online exercise and question $\&$ answer: Students participating in network teaching can accomplish online exercises and examinations independently, and the system can provide scores in accordance with standard answers. The website also sets a special communication region for questions and answers between students and teachers.

\subsection{Teaching effect of virtual surveying experimental system}

Establishing various experimental courses oriented to surveying major: The established virtual simulation platform has various experimental programs, from demonstration verification to design innovation. The virtual simulation experimental teaching system is composed of 6 curriculum groups and 15 experimental courses and serves for 14 majors of 9 colleges.

Significant improvement in students' practice and innovation ability: The virtual experimental platform and training can significantly improve students' practice and innovation abilities. A total of 60 person-times have won awards in national and provincial undergraduate surveying skill competitions, the statistical results are shown in Table 1[12], and 15 projects have won awards in national Challenge Cup, National University GIS Competition of Super Map Cup, and Chinese Undergraduate GIS Software Development of ESRI Cup. Three theses have been evaluated as the Undergraduate Dissertation of Henan Province. Three items have gained patents and four items have won software copyrights. Six academic papers have been published. These achievements reflect the improved students' practice and innovation abilities. 
Table 1. Statistics on different levels of surveying skills competitions in recent years of Henan University of Urban Construction

\begin{tabular}{|c|c|c|c|}
\hline No. & Competition category & Item & Awards \\
\hline \multirow{4}{*}{1} & \multirow{4}{*}{$\begin{array}{l}\text { 4th National Undergraduate Surveying } \\
\text { Skill Competition } \\
\text { August, } 2016\end{array}$} & Traverse survey & Grand prize \\
\hline & & Fourth-class leveling & First prize \\
\hline & & Digital surveying & Second prize \\
\hline & & Programming & First prize \\
\hline \multirow{3}{*}{2} & \multirow{3}{*}{$\begin{array}{l}\text { 3rd National Undergraduate Surveying } \\
\text { Skill Competition } \\
\text { July, } 2014\end{array}$} & Traverse survey & First prize \\
\hline & & Fourth-class leveling & First prize \\
\hline & & Digital surveying & First prize \\
\hline \multirow{4}{*}{3} & \multirow{4}{*}{$\begin{array}{l}\text { 4th Henan Undergraduate Surveying Skill } \\
\text { Competition } \\
\text { May, } 2016\end{array}$} & Traverse survey & First prize \\
\hline & & Fourth-class leveling & Second prize \\
\hline & & Digital surveying & Grand prize \\
\hline & & Programming & First prize \\
\hline \multirow{3}{*}{4} & \multirow{3}{*}{$\begin{array}{l}\text { 3rd Henan Undergraduate Surveying Skill } \\
\text { Competition } \\
\text { May, } 2014\end{array}$} & Traverse survey & First prize \\
\hline & & Fourth-class leveling & First prize \\
\hline & & Digital surveying & First prize \\
\hline
\end{tabular}

\section{Conclusions}

An experimental virtual surveying system was designed using VR technology to address the issue of bottlenecks of traditional experimental surveying teaching in equipment, sites, time, and environment, as well as provide students with a comprehensive and unlimited teaching platform for surveying. This system was implemented in the experimental teaching of surveying courses in Henan University of Urban Construction. The following conclusions were derived.

1. The learning process of students was not restricted by time and sites with their use of the experimental network virtual surveying system. Thus, the experimental cost was reduced and the experimental efficiency was enhanced.

2. The online lectures and instrument virtualization components of the proposed system provided students with extensive self-learning opportunities, guided them to learn autonomously by combining theories and practice, and enhanced their innovation abilities.

3. The simulation of large surveying engineering project provided hands-on training to students in large projects and improved their practical and project management skills.

The development of a virtual experimental system provides a novel experimental teaching mode for surveying courses. However, this comprehensive engineering system has to combine the characteristics and development trend of surveying engineering majors to promote the continuous research and development, utilization, and improvement of experimental virtual surveying systems. 


\section{References}

[1] Yang Min, Wang Yunjia, Yu Jieqian and Guo Guangli. Innovation and exploration on construction of virtual simulation experiment teaching center of mine surveying,Mine Surveying, 2016, vol. 44(4), pp. 98-102.

[2] Tian Peng, Wang Yunjia, Wang Yong, Song Zhiming. Design and implementation of virtual simulation teaching system for total station instrument[J].Journal of Huaihai Institute of Technology(Natural Science Edition), 2016, vol. 25(4), pp. 43-47.

[3] Koncny M. Cartography:Challenges and potential in the virtual Geographic environments era[J].Annals of GIS, 2011, vol. 17(3), pp. 135-145. https://doi.org/10.1080/1947568 $\underline{3.2011 .602027}$

[4] Lin Hui,Chen Min.Experimental geography based on virtual geographic environments.Geomatics and Information Science of Wuhan University, 2014, vol. 39(6), pp. 689-694.

[5] Zhou Min, Fan Cong. Design and implementation of virtual measurement experiment based on unity3d.Geomatics \& Spatial Information Technology, 2016, vol. 39 (1), pp. $179-181$.

[6] Deng Xingsheng. Research on photogrammetry practice teaching pattern based on virtual reality platform .Engineering of Surveying and Mapping, 2015, vol. 24(9) , pp. 74-76.

[7] Wang Shitai, Yin Min.Research on the use of virtual reality technology in practical teaching of surveying.Geomatics \& Spatial Information Technology, 2012,35(1), pp. 222-224.

[8] Zang Jie, Li Rong, Zhao Yunchang, and Yang Zhao. Application of virtual training system to the automobile mechanical engineering experimental teaching,Journal of Heilongjiang Institute of Technology, 2016, vol. 30(3), pp. 57-62 .

[9] Wang Panpan,Zhang Heng. Design of virtual measuring system based on tools ,Experimental Technology and Management, 2015, vol. 32(3) , pp. 148-151.

[10] Li Ping, Mao Changjie, Xu Jin. Construction of the national virtual simulation experiment teaching centers, improving the experiment teaching informatization in higher education, Research and Exploration in Laboratory, 2013, vol. 32(11), pp. 5-8.

[11] He Junliang, Zhang Bing, Sun Zhongwei, Zhao Xuyang. Construction and release of virtual scenes based on 3D GIS,Science of Surveying and Mapping, 2013, vol. 38(4) , pp. 172174.

[12] Gao Ning,Gao Caiyun.The use of comprehensive practical skill competitions in cultivating the innovative abilities of surveying undergraduates.World Transactions on Engineering and Technology Education, 2015, vol. 13(4), pp. 469-473.

\section{$7 \quad$ Authors}

Caiyun Gao is a lecturer in School of Geomatics \& Urban Information, Henan University of Urban Construction, Pingdingshan 467036, China (gaocaiyungao@163.com).

Qiutang Wu is an engineer in Basic Surveying and Mapping Institute of Jiangxi Province, Nanchang, 330000, China (249630128@qq.com).

Article submitted 18 March 2017. Published as resubmitted by the authors 19 April 2017. 